PROCEEDINGS OF THE

AMERICAN MATHEMATICAL SOCIETY

Volume 131, Number 9, Pages 2937-2944

S 0002-9939(03)06842-4

Article electronically published on January 8, 2003

\title{
A STRONG LAW OF LARGE NUMBERS FOR GENERALIZED RANDOM SETS FROM THE VIEWPOINT OF EMPIRICAL PROCESSES
}

\author{
FRANK N. PROSKE AND MADAN L. PURI
}

(Communicated by Claudia M. Neuhauser)

\begin{abstract}
In this article we prove a strong law of large numbers for Borel measurable nonseparably valued random elements in the case of generalized random sets.
\end{abstract}

\section{INTRODUCTION}

In this paper we provide a proof of the strong law of large numbers (SLLN) for generalized random sets in the case of pairwise independent and identically distributed fuzzy random variables, extending similar results for random sets in a natural way (see e.g. Artstein and Vitale [1], Giné, Hahn and Zinn [6] and Puri and Ralescu [12]). Here, we deal with the concept of a fuzzy random variable introduced by Puri and Ralescu [13].

Fuzzy random variables are suitable for describing random experiments whose outcomes are due to imprecision of human knowledge. This kind of nonstatistical inexactness occurs e.g. in the form of linguistic terms which can be represented by fuzzy sets.

In section 2 we record some facts about random sets, fuzzy random variables and other preliminaries, which are required for the proof of our main result, a strong law of large numbers for fuzzy random variables, being tackled in section 3. Here, we choose the framework of $R^{p}$-valued fuzzy random variables, although our method of proof is applicable to the case of separable Banach spaces.

In literature, strong laws of large numbers for fuzzy random variables are already established: Klement, Puri and Ralescu [8] proved such a result, but using two different metrics: The Borel measurability of a fuzzy random variable is defined via one metric, whereas the convergence is induced by the other. Their approach relies on embedding theorems and results from probability on Banach spaces. A stronger convergence result for independent and pairwise identically distributed fuzzy random variables could be shown by Kruse [9] for $R$, utilizing its total order.

In handling the nonseparability of involved metric spaces of fuzzy sets, the main tool of our proof is to associate linear isometrically each convex fuzzy random variable with an empirical process in $l^{\infty}(T)$, i.e. a Glivenko-Cantelli problem is set

Received by the editors March 12, 2002 and, in revised form, April 11, 2002.

2000 Mathematics Subject Classification. Primary 60D05; Secondary $03 E 72$.

Key words and phrases. Random set, fuzzy set, fuzzy random variable, embedding, Hausdorff distance, empirical process, strong law of large numbers. 
(see e.g. Hoffmann-Jørgensen [7] and Talagrand [14]). In solving it, we verify a certain finiteness condition of entropy with bracketing for the resulting empirical process.

Artstein and Vitale [1] proved a SLLN for $R^{p}$-valued random sets with respect to the Hausdorff distance (i.e. random sets whose values are compact subsets of $\left.R^{p}\right)$. Later on this result was extended by Giné, Hahn and Zinn [6] and Puri and Ralescu 13 to separable Banach spaces.

Regarding the finite-dimensional case, our result is a natural generalization of all previous SLLN's for random sets, because Borel measurability and convergence is only based upon one metric which generalizes the Hausdorff distance.

In the sequel we assume a probability space $(\Omega, \Im, P)$ to be complete.

\section{Preliminaries}

Let $\left(R^{p},\|\cdot\|\right)$ be the Euclidean space.

A fuzzy set of $R^{p}$ is a function $u: R^{p} \longrightarrow[0,1]$. For each fuzzy set $u$ the $\alpha$-level set is defined by $L_{\alpha} u:=\left\{x \in R^{p} \mid u(x) \geq \alpha\right\}, 0<\alpha \leq 1$. By supp $u$ we understand the closure of $\left\{x \in R^{p} \mid u(x)>0\right\}$ in $R^{p}$. We denote by $F_{c}\left(R^{p}\right)$ the collection of those fuzzy sets $u: R^{p} \longrightarrow[0,1]$ which have the following properties: (i) $L_{\alpha} u$ is compact for all $0<\alpha \leq 1$; (ii) $\operatorname{supp} u$ is compact; (iii) $\left\{x \in R^{p} \mid u(x)=1\right\} \neq \varnothing$.

In the sequel we set $L_{0} u=\operatorname{supp} u$. Zadeh's Extension principle [16] supplies a linear structure in $F_{c}\left(R^{p}\right)$ by the operations

$$
\begin{aligned}
(u+v)(x) & :=\sup _{y+z=x} \min (u(y), v(z)), \\
(\lambda u)(x) & := \begin{cases}u\left(\lambda^{-1} x\right), & \text { if } \lambda \neq 0, \\
\chi_{\{0\}}(x), & \text { if } \lambda=0,\end{cases} \\
\text { for } u, v & \in F_{c}\left(R^{p}\right), \lambda \in R .
\end{aligned}
$$

For later use, we define $U_{c}\left(R^{p}\right)$ as the collection of all $u \in F_{c}\left(R^{p}\right)$ with convex $\alpha$-level sets and denote the convex hull of $u \in F_{c}\left(R^{p}\right)$ by co $u \in F_{c}\left(R^{p}\right)$, determined by its convex compact $\alpha$-level sets $L_{\alpha} \operatorname{cou}:=\operatorname{co}\left(L_{\alpha} u\right), 0<\alpha \leq 1$ (see Klement, Puri and Ralescu [8]), where co $A$ is the closure of the convex hull of a subset $A \subseteq R^{p}$ in $R^{p}$.

Denote by $\mathcal{K}\left(R^{p}\right)\left(\operatorname{co} \mathcal{K}\left(R^{p}\right)\right)$, the class of all nonempty (convex and) compact subsets of $R^{p}$. The space $\mathcal{K}\left(R^{p}\right)\left(\operatorname{co} \mathcal{K}\left(R^{p}\right)\right)$ together with the following operations forms a linear abelian semigroup:

$$
\begin{aligned}
& \quad A+B:=\{a+b \mid a \in A, b \in B\}, \lambda A:=\{\lambda a \mid a \in A\}, \\
& \text { for } A, B \in \mathcal{K}\left(R^{p}\right)\left(\operatorname{co} \mathcal{K}\left(R^{p}\right)\right), \lambda \in R .
\end{aligned}
$$

Then the following properties can be shown, which are needed later (see Puri and Ralescu [12], [13]):

$$
L_{\alpha}(u+v)=L_{\alpha} u+L_{\alpha} v, L_{\alpha}(\lambda u)=\lambda L_{\alpha} u
$$

and

$$
c o\left(L_{\alpha} u+L_{\alpha} v\right)=c o L_{\alpha} u+c o L_{\alpha} v, c o\left(\lambda L_{\alpha} u\right)=\lambda c o L_{\alpha} u
$$

for all $u, v \in F_{c}\left(R^{p}\right), 0<\alpha \leq 1$. Note also that $F_{c}\left(R^{p}\right)$ and $U_{c}\left(R^{p}\right)$ are closed under the operations above and form, like $\mathcal{K}\left(R^{p}\right)$ or $\operatorname{co} \mathcal{K}\left(R^{p}\right)$, a linear abelian semigroup. 
$\mathcal{K}\left(R^{p}\right)$ is metrizable by the well-known Haudorff distance $d_{H}$, given by $d_{H}: \mathcal{K}\left(R^{p}\right) \times \mathcal{K}\left(R^{p}\right) \longrightarrow[0, \infty) ;(A, B) \mapsto \max \left\{\sup _{a \in A} \inf _{b \in B}\|a-b\|, \sup _{b \in B} \inf _{a \in A}\|a-b\|\right\}$. $\left(\mathcal{K}\left(R^{p}\right), d_{H}\right)$ and $\left(\operatorname{co} \mathcal{K}\left(R^{p}\right), d_{H}\right)$ are then complete separable metric spaces (see Puri and Ralescu [13]). By identifying each $A \in \mathcal{K}\left(R^{p}\right)$ with its characteristic function $\chi_{A} \in F_{c}\left(R^{p}\right)$ we can generalize the Hausdorff distance to the case of $F_{c}\left(R^{p}\right)$, getting a continuous semigroup monomorphism:

Proposition 1 (see Puri and Ralescu [13]). $\left(F_{c}\left(R^{p}\right), d_{\infty}\right)\left(\left(U_{c}\left(R^{p}\right), d_{\infty}\right)\right)$ is a complete, but in contrast to $\left(\mathcal{K}\left(R^{p}\right), d_{H}\right)\left(\left(\right.\right.$ co $\left.\left.\mathcal{K}\left(R^{p}\right), d_{H}\right)\right)$ a nonseparable metric space, endowed with the metric $d_{\infty}$ defined by $d_{\infty}(u, v)=\sup _{0<a \leq 1} d_{H}\left(L_{\alpha} u, L_{\alpha} v\right)$ for $u, v \in F_{c}\left(R^{p}\right)$.

Let $(\Omega, \Im, P)$ be a probability space. A fuzzy random variable (frv) is a Borel measurable function $\xi: \Omega \longrightarrow\left(F_{c}\left(R^{p}\right), d_{\infty}\right)$. As one can see, frvs generalize compact random sets $A$ (i.e. Borel measurable functions $A: \Omega \longrightarrow\left(\mathcal{K}\left(R^{p}\right), d_{H}\right)$ ) by identification with the characteristic function $\chi_{A}$.

Proposition 2 (see Klement, Puri and Ralescu [8]). Let $\xi: \Omega \longrightarrow\left(F_{c}\left(R^{p}\right), d_{\infty}\right)$ be a frv. Then

(i) $\operatorname{co} \xi: \Omega \longrightarrow\left(U_{c}\left(R^{p}\right), d_{\infty}\right) ; \omega \mapsto \operatorname{co} \xi(\omega)$ is a frv.

(ii) $L_{\alpha} \xi: \Omega \longrightarrow\left(\mathcal{K}\left(R^{p}\right), d_{H}\right) ; \omega \mapsto L_{\alpha} \xi(\omega)$ and $\operatorname{co}\left(L_{\alpha} \xi\right): \Omega \longrightarrow\left(\operatorname{co} \mathcal{K}\left(R^{p}\right), d_{H}\right)$; $\omega \longmapsto \operatorname{co}\left(L_{\alpha} \xi(\omega)\right)$ are (convex) compact random sets for all $0<\alpha \leq 1$.

(iii) $\operatorname{supp} \xi: \Omega \longrightarrow\left(\mathcal{K}\left(R^{p}\right), d_{H}\right) ; \omega \mapsto L_{0} \xi(\omega)$ is a compact random set.

Proposition 3 (see Puri and Ralescu [14]). Let $\xi: \Omega \longrightarrow\left(F_{c}\left(R^{p}\right), d_{\infty}\right)$ be a frv with $E\|\xi\|_{\infty}<\infty\left(\|\xi(\omega)\|_{\infty}:=\sup _{\alpha>0} d_{H}\left(\{0\}, L_{\alpha} \xi(\omega)\right)\right)$. Then there exists a unique fuzzy set $u \in U_{c}\left(R^{p}\right)$ such that the $\alpha$-level sets of $u$ are the Aumann expectations of the convex compact random sets $\operatorname{co}\left(L_{\alpha} \xi\right)$ (for definition, see Aubin and Frankowska 2], Debreu [4]), i.e. $L_{\alpha} u=E \operatorname{co}\left(L_{\alpha} \xi\right)$ for all $0<\alpha \leq 1$.

If $\xi: \Omega \longrightarrow\left(F_{c}\left(R^{p}\right), d_{\infty}\right)$ is a frv with $E\|\xi\|_{\infty}<\infty$ then, by the last result, the expected value $E \xi$ is the unique convex fuzzy set satisfying the property $L_{\alpha} E \xi=$ $E \operatorname{co}\left(L_{\alpha} \xi\right), 0<\alpha \leq 1$.

A convenient representation of convex compact sets as elements of a concrete Banach space involves using support functions. Let us denote by ball $R^{p}$, the compact unit ball of $R^{p}$. The support function of a convex compact subset $A \subseteq R^{p}$ is the function $s_{A}$ defined on ball $R^{p}$ by the equation

$$
s_{A}(x):=\sup _{a \in A}\langle x, a\rangle, x \in \text { ball } R^{p} .
$$

A crucial step in the proof of the main theorem is based on an embedding of convex compact sets into the space of continuous functions. This result goes back to Minkowski (see e.g. Artstein and Vitale [1]) :

Theorem 4. The function $j:\left(\operatorname{co} \mathcal{K}\left(R^{p}\right), d_{H}\right) \longrightarrow\left(C\left(\right.\right.$ ball $\left.\left.R^{p},\|\cdot\|\right),\|\cdot\|_{\infty}\right)\left(\|\cdot\|_{\infty}\right.$ is the supremum norm) given by $A \longmapsto\left(x \mapsto s_{A}(x)\right)$ satisfies

(i) $j$ is an isometry (i.e. $\left.d_{H}(A, B)=\|j(A)-j(B)\|_{\infty}\right)$.

(ii) $j(A+B)=j(A)+j(B), j(\lambda A)=\lambda j(A)(\lambda \geq 0)$.

A central idea in deriving a SLLN for $F_{c}\left(R^{p}\right)$-valued frvs (see Corollary 7 ) is to reduce first the study of frvs to the convex case by convexifying the underlying 
variables and then to get rid of the convexity restriction by applying the following inequality (see e.g. Giné, Hahn and Zinn [6]):

Theorem 5 (Shapley-Folkman-Starr). We obtain for all $A_{1}, \ldots, A_{n} \in \mathcal{K}\left(R^{p}\right)$ and $n \in I N$ :

$$
d_{H}\left(A_{1}+\ldots+A_{n}, \operatorname{co}\left(A_{1}+\ldots+A_{n}\right)\right) \leq \sqrt{p} \max _{1 \leq i \leq n}\left\|A_{i}\right\|\left(\|A\|:=d_{H}(\{0\}, A)\right) .
$$

The proof of the main result requires the notion of a bracketing number to measure the "size" of certain function classes: Let $(\mathcal{F},\|\cdot\|)$ be a subset of a normed space of real functions $f: \chi \longrightarrow R$ on a set $\chi$. Then the bracket $[l, u]$ for real functions $l, u$ is the set of all functions $f: \chi \longrightarrow R$ satisfying $l \leq f \leq u$. An $\varepsilon$-bracket is a bracket $[l, u]$ with $\|l-u\|<\varepsilon$. Then the bracketing number $N_{[]}(\varepsilon, \mathcal{F},\|\cdot\|)$ is the minimum number of $\varepsilon$-brackets covering $\mathcal{F}$. Note that in the definition of the bracketing number the functions $l$ and $u$ are assumed to have finite norms, but not to belong to $\mathcal{F}$ itself.

\section{Strong LAW OF LARGE NUMBERS FOR FUZZY RANDOM VARIABLES}

First, we give a Kolmogorov type of SLLN for convex frvs:

Theorem 6. If $\xi_{i}: \Omega \longrightarrow\left(U_{c}\left(R^{p}\right), d_{\infty}\right)$ is a p.i.i.d. sequence of fros on the probability space $(\Omega, \Im, P)$ with $E\left\|\xi_{1}\right\|_{\infty}<\infty$, then

$$
d_{\infty}\left(\frac{1}{n} \sum_{i=1}^{n} \xi_{i}, E \xi_{1}\right) \longrightarrow 0 \text { a.e. }
$$

Proof. We intend to solve a Glivenko-Cantelli problem for the function class $\mathcal{F}:=$ $\left\{f_{t} \mid t \in T\right\}$ consisting of $\mathcal{B}\left(U_{c}\left(R^{p}\right)\right), \mathcal{B}(R)$-measurable functions $f_{t}:\left(U_{c}\left(R^{p}\right), d_{\infty}\right)$ $\longrightarrow R ; u \longmapsto s_{L_{\alpha} u}(x)$ for $t=(\alpha, x) \in T:=[0,1] \times$ ball $R^{p}$. For that purpose, it is sufficient to check a finiteness condition of entropy with bracketing, i.e. we should prove that $N\left(\varepsilon, \mathcal{F}, L_{1}(P)\right)<\infty$ holds for all $\varepsilon>0$, where $L_{1}(P)$ denotes the usual $L_{1}$-norm with respect to $P$. We subdivide the proof into several steps:

1. Let us equip the interval $[0,1]$ with the pseudodistance

$$
\rho_{1}:[0,1] \times[0,1] \longrightarrow[0, \infty) ;\left(\alpha_{1}, \alpha_{2}\right) \mapsto d_{H}\left(L_{\alpha_{1}} E \xi_{1}, L_{\alpha_{2}} E \xi_{1}\right) .
$$

Since the $\alpha$-level sets of a fuzzy set $u \in F_{c}\left(R^{p}\right)$ are nonincreasing and since the mapping $\left(\alpha \mapsto L_{\alpha} u\right)$ on the interval $[0,1]$ is left continuous in $(0,1]$ as well as right continuous at 0 with respect to the Hausdorff distance (see Klement, Puri and Ralescu [8]), one realizes that the $\rho_{1}$-balls with radius $<\varepsilon$ and centers $0 \leq \alpha_{0} \leq 1$, $U_{\varepsilon}^{\left(\rho_{1}\right)}\left(\alpha_{0}\right)$, are nondegenerate subintervals of $[0,1]$.

2. For our convenience we denote $f_{t}(\cdot)=f((\alpha, x), \cdot)$ for $t=(\alpha, x)$. We find that

$$
\left|f\left(\left(\alpha_{2}, x_{1}\right), \xi_{1}\right)-f\left(\left(\alpha_{2}, x_{2}\right), \xi_{1}\right)\right| \leq\left\|\xi_{1}\right\|_{\infty}\left\|x_{1}-x_{2}\right\|
$$

holds for all $\left(\alpha_{2}, x_{1}\right),\left(\alpha_{2}, x_{2}\right) \in T$. This leads to the following inequality:

$$
\begin{aligned}
A & :=\left|f\left(\left(\alpha_{1}, x_{1}\right), \xi_{1}\right)-f\left(\left(\alpha_{2}, x_{2}\right), \xi_{1}\right)\right| \\
& \leq\left(\left|f\left(\left(\alpha_{1}, x_{1}\right), \xi_{1}\right)-f\left(\left(\alpha_{2}, x_{1}\right), \xi_{1}\right)\right|+\left\|\xi_{1}\right\|_{\infty}\left\|x_{1}-x_{2}\right\|\right)
\end{aligned}
$$

for $\left(\alpha_{1}, x_{1}\right),\left(\alpha_{2}, x_{2}\right) \in T$. 
We go on with bounding above and obtain

$$
\begin{aligned}
\|A\|_{P, 1} & =E\left|f\left(\left(\alpha_{1}, x_{1}\right), \xi_{1}\right)-f\left(\left(\alpha_{2}, x_{2}\right), \xi_{1}\right)\right| \\
& \leq\left(\sup _{x \in \text { ball } R^{p}} E\left|f\left(\left(\alpha_{1}, x\right), \xi_{1}\right)-f\left(\left(\alpha_{2}, x\right), \xi_{1}\right)\right|+\left(E\left\|\xi_{1}\right\|_{\infty}\right)\left\|x_{1}-x_{2}\right\|\right) \\
& \leq K\left(\sup _{x \in \text { ball } R^{p}} E\left|f\left(\left(\alpha_{1}, x\right), \xi_{1}\right)-f\left(\left(\alpha_{2}, x\right), \xi_{1}\right)\right|+\left\|x_{1}-x_{2}\right\|\right) \\
& =K \rho\left(\left(\alpha_{1}, x_{1}\right),\left(\alpha_{2}, x_{2}\right)\right) \text { for all }\left(\alpha_{1}, x_{1}\right),\left(\alpha_{2}, x_{2}\right) \in T
\end{aligned}
$$

with the pseudodistance $\rho=\rho_{1}+\rho_{2}$ on $T$ where $\rho_{2}=\|\cdot\|$.

The last relation is a consequence of Theorem 4, and of the fact that $E s_{A}(x)=$ $s_{E A}(x)\left(x \in R^{p}\right)$ is valid for the Aumann expectation of compact random sets $A$ (see Aubin and Frankowska [2]).

3. We plan to construct a finite $\varepsilon-$ net for the pseudodistance $\rho_{1}$.

For that purpose, let us define: $\alpha_{0}:=1$,

$\alpha_{1}:=\inf \left\{\alpha \in[0,1] \mid \alpha \leq \alpha_{0}, \rho_{1}\left(\alpha, \alpha_{0}\right)<\varepsilon\right\}$.

If $\alpha_{1}=0$, cut short the procedure; otherwise define:

$\alpha_{2}:=\inf \left\{\alpha \in[0,1] \mid \alpha \leq \alpha_{1}, \rho_{1}\left(\alpha, \alpha_{1}\right)<\varepsilon\right\}$

:

continuing by induction.

Suppose now that the procedure does not stop.

The left-continuity of $\left(\alpha \mapsto \rho_{1}\left(\alpha, \alpha_{n-1}\right)\right)$ on $(0,1]$ for all $n$ implies then $\rho_{1}\left(\alpha_{n}, \alpha_{n-1}\right) \geq \varepsilon$ for all $n$. Because of the compactness of $L_{\alpha_{n}} E \xi_{1}$ and the continuity of $\left(x \mapsto \inf _{y \in L_{\alpha_{n-1}} E \xi_{1}}\|x-y\|\right)$ for all $n$, there exists a sequence $\left(a_{n}\right)_{n \in I N}$ in $\operatorname{supp} E \xi_{1}$ such that $\left\|a_{i}-a_{j}\right\| \geq \varepsilon$ for $i \neq j$. But this is in contradiction with the compactness of $\operatorname{supp} E \xi_{1}$.

To sum up, there exists a natural number $n$ such that $\alpha_{0}=1, \alpha_{n}=0, \alpha_{m}<$ $\alpha_{m-1}$ and $\rho_{1}\left(\alpha, \alpha_{m-1}\right)<\varepsilon$ on $\left(\alpha_{m}, \alpha_{m-1}\right]$ for all $m=1, \ldots, n$. Thus $\left(\alpha_{m}\right)_{m=0}^{n}$ is a $\varepsilon-$ net for $\rho_{1}$. Since (ball $\left.R^{p},\|\cdot\|\right)$ is a compact metric space, we conclude in addition that there is a finite $\varepsilon-$ net for $\rho$.

4. From step 3 we know that there exists a finite $(\rho-\varepsilon)-\operatorname{net}\left(S_{i}^{(\varepsilon)}\right)_{i=1}^{n}$. Since $U_{\varepsilon}^{(\rho)}\left(S_{i}^{(\varepsilon)}\right)$ is a subset of $U_{\varepsilon}^{\left(\rho_{1}\right)}\left(\alpha_{i}^{(\varepsilon)}\right) \times U_{\varepsilon}^{\left(\rho_{2}\right)}\left(x_{i}^{(\varepsilon)}\right)$ for $S_{i}^{(\varepsilon)}=\left(\alpha_{i}^{(\varepsilon)}, x_{i}^{(\varepsilon)}\right), i=1, \ldots, n$, we get

$$
\begin{aligned}
C:= & \sup _{\substack{(\alpha, x) \in \\
U_{\varepsilon}^{(\rho)}\left(S_{i}^{(\varepsilon)}\right)}}\left|f\left((\alpha, x), \xi_{1}\right)-f\left(\left(\alpha_{i}^{(\varepsilon)}, x_{i}^{(\varepsilon)}\right), \xi_{1}\right)\right| \\
\leq & (\underbrace{\sup _{\alpha \in U_{\varepsilon}^{\left(\rho_{1}\right)}\left(\alpha_{i}^{(\varepsilon)}\right)}\left|f\left(\left(\alpha, x_{i}^{(\varepsilon)}\right), \xi_{1}\right)-f\left(\left(\alpha_{i}^{(\varepsilon)}, x_{i}^{(\varepsilon)}\right), \xi_{1}\right)\right|}_{:=f(\varepsilon)} \\
& \left.+\left\|\xi_{1}\right\|_{\infty} \sup _{x \in U_{\varepsilon}^{\left(\rho_{2}\right)}\left(x_{i}^{(\varepsilon)}\right)}\left\|x-x_{i}^{(\varepsilon)}\right\|\right) .
\end{aligned}
$$

Denote

$$
C_{1}^{(n)}\left|f\left(\left(\underline{\alpha}_{n}, x_{i}^{(\varepsilon)}\right), \xi_{1}\right)-f\left(\left(\alpha_{i}^{(\varepsilon)}, x_{i}^{(\varepsilon)}\right), \xi_{1}\right)\right|
$$

and

$$
C_{2}^{(n)}\left|f\left(\left(\bar{\alpha}_{n}, x_{i}^{(\varepsilon)}\right), \xi_{1}\right)-f\left(\left(\alpha_{i}^{(\varepsilon)}, x_{i}^{(\varepsilon)}\right), \xi_{1}\right)\right|
$$


Because of step 1 and monotonicity reasons there exist sequences $\underline{\alpha}_{n} \searrow$ and $\bar{\alpha}_{n} \nearrow$ independent of $\omega \in \Omega$ and of $U_{\varepsilon}^{\left(\rho_{1}\right)}\left(\alpha_{i}^{(\varepsilon)}\right)$ such that

$$
f(\varepsilon)=\max \left\{\lim _{n} C_{1}^{(n)}, \lim _{n} C_{2}^{(n)}\right\} .
$$

Now, using the monotone convergence theorem and step 2, we realize

$$
\begin{aligned}
E f(\varepsilon) & =\lim _{n} E \max \left\{C_{1}^{(n)}, C_{2}^{(n)}\right\} \\
& \leq \lim _{n}\left(E C_{1}^{(n)}+E C_{2}^{(n)}\right) \\
& \leq \lim _{n} K\left(\rho_{1}\left(\underline{\alpha}_{n}, \alpha_{i}^{(\varepsilon)}\right)+\rho_{1}\left(\bar{\alpha}_{n}, \alpha_{i}^{(\varepsilon)}\right)\right) \\
& \leq \widetilde{K} \varepsilon .
\end{aligned}
$$

Since the continuity of the expressions $\inf _{t \in A} f_{t}(\cdot)$ and $\sup _{t \in A} f_{t}(\cdot)$ for $A \subseteq T$ guarantees the measurability of $C$, we can write

$$
E C \leq\left(E f(\varepsilon)+\left(E\left\|\xi_{1}\right\|_{\infty}\right) \varepsilon\right) \leq K_{0} \varepsilon
$$

5. First, we want to find a finite number of $\varepsilon$-brackets which cover the function class $\mathcal{F}=\left\{f_{t} \mid t \in T\right\}$. While doing so, let us define for $t_{0} \in T$ and $\varepsilon>0$, $f_{\varepsilon, t_{0}}^{u}:=\sup _{t \in U_{\varepsilon}^{(\rho)}\left(t_{0}\right)} f_{t}(\cdot)$ and $f_{\varepsilon, t_{0}}^{l}:=\inf _{t \in U_{\varepsilon}^{(\rho)}\left(t_{0}\right)} f_{t}(\cdot)$. For the same reasons, mentioned at the end of step $4, f_{\varepsilon, t_{0}}^{u}$ and $f_{\varepsilon, t_{0}}^{l}$ are measurable functions. Because of step 3, there exists a finite $(\rho-\varepsilon)-$ net, $S(\varepsilon) \subseteq T$. We see then that for all $t \in T$ there is a $s \in S(\varepsilon)$ such that for all $v \in U_{c}\left(R^{p}\right), f_{\varepsilon, s}^{l}(v) \leq f_{t}(v) \leq f_{\varepsilon, s}^{u}(v)$ holds.

Furthermore, using the last inequality of step 4 , we realize that

$$
\begin{aligned}
& \left\|f_{\varepsilon, s}^{u}-f_{\varepsilon, s}^{l}\right\|_{P, 1} \\
& =E\left(f_{\varepsilon, s}^{u}\left(\xi_{1}\right)-f_{\varepsilon, s}^{l}\left(\xi_{1}\right)\right) \\
& =E\left(\sup _{t_{1}, t_{2} \in U_{\varepsilon}^{(\rho)}(s)}\left|f_{t_{1}}\left(\xi_{1}\right)-f_{t_{2}}\left(\xi_{1}\right)\right|\right) \\
& \leq 2 E\left(\sup _{t \in U_{\varepsilon}^{(\rho)}(s)}\left|f_{t}\left(\xi_{1}\right)-f_{s}\left(\xi_{1}\right)\right|\right) \\
& \leq K \varepsilon \text { for all } s \in S(\varepsilon) \text { and a constant } K .
\end{aligned}
$$

Putting everything together, we could show that

$$
N_{[]}\left(\varepsilon, \mathcal{F}, L_{1}(P)\right)<\infty \text { holds for all } \varepsilon>0 .
$$

To finish the proof, pick up finitely many $\varepsilon$-brackets $\left[l_{j}, u_{j}\right]$, whose union covers $\mathcal{F}=\left\{f_{t} \mid t \in T\right\}$. Then, for every $t \in T$, there exists a bracket such that

$$
\begin{aligned}
\frac{1}{n} \sum_{i=1}^{n} f_{t}\left(\xi_{i}\right)-E f_{t}\left(\xi_{1}\right) & \leq \frac{1}{n} \sum_{i=1}^{n} u_{j}\left(\xi_{i}\right)-E u_{j}\left(\xi_{1}\right)+E\left(u_{j}\left(\xi_{1}\right)-f_{t}\left(\xi_{1}\right)\right) \\
& \leq \frac{1}{n} \sum_{i=1}^{n} u_{j}\left(\xi_{i}\right)-E u_{j}\left(\xi_{1}\right)+\varepsilon .
\end{aligned}
$$

Therefore, we find

$$
\sup _{t \in T}\left(\frac{1}{n} \sum_{i=1}^{n} f_{t}\left(\xi_{i}\right)-E\left(\xi_{1}\right)\right) \leq \max _{j}\left(\frac{1}{n} \sum_{i=1}^{n} u_{j}\left(\xi_{i}\right)-E u_{j}\left(\xi_{1}\right)\right)+\varepsilon .
$$


The last term of the above inequality tends almost surely to $\varepsilon$ by Etemadi's strong law of large numbers (see Etemadi [5]). Arguing in a similar way for the infimum on the left side, we obtain together with Theorem 4

$$
\lim \sup d_{\infty}\left(\frac{1}{n} \sum_{i=1}^{n} \xi_{i}, E \xi_{1}\right) \leq \varepsilon \text { a.e. }
$$

implying the desired result.

The SLLN is even valid for $F_{c}\left(R^{p}\right)$-valued frvs:

Corollary 7. Let $\xi_{i}: \Omega \longrightarrow\left(F_{c}\left(R^{p}\right), d_{\infty}\right)$ be a p.i.i.d. sequence of frvs with $E\left\|\xi_{1}\right\|_{\infty}<\infty$; then

$$
d_{\infty}\left(\frac{1}{n} \sum_{i=1}^{n} \xi_{i}, E \xi_{1}\right) \longrightarrow 0 \text { a.e. }
$$

Proof. Let us first pay our attention to the p.i.i.d. frvs $\operatorname{co} \xi_{i}: \Omega \longrightarrow\left(U_{c}\left(R^{p}\right), d_{\infty}\right)$. The Shapley-Folkman-Starr theorem supplies:

$$
d_{\infty}\left(\sum_{i=1}^{n} \xi_{i} / n, \sum_{i=1}^{n} \operatorname{co} \xi_{i} / n\right) \leq \sqrt{p} \max _{1 \leq i \leq n}\left\|\xi_{i}\right\|_{\infty} / n .
$$

We can then write

$$
\begin{aligned}
& d_{\infty}\left(\sum_{i=1}^{n} \xi_{i} / n, E \xi_{1}\right) \\
& \quad \leq \sqrt{p} \max _{1 \leq i \leq n}\left\|\xi_{i}\right\|_{\infty} / n+d_{\infty}\left(\sum_{i=1}^{n} \operatorname{co} \xi_{i} / n, E \xi_{1}\right) .
\end{aligned}
$$

The first term of the above inequality converges a.e. to zero (see, for example, p. 122 in Chow and Teicher [3]). The same is true of the second by virtue of the last theorem.

\section{Conclusion}

Our main result generalizes in a natural way that of Klement, Puri and Ralescu [8], even for pairwise independent and identically distributed frvs. It seems to be that our method of proof is also available for proving a law of iterated logarithm for frvs.

\section{REFERENCES}

1. Artstein, Z. and Vitale, R.A. (1975). A strong law of large numbers for random compact sets. Ann. Prob. 3, 879-882. MR 52:6825

2. Aubin, J.P. and Frankowska, H. (1990). Set-valued Analysis. Systems \& Control: Foundations \& Applications, Birkhäuser. MR 91d:49001

3. Chow, Y.S. and Teicher, H. (1978). Probability theory. Springer. MR 80a:60004

4. Debreu, G. (1966). Integration of correspondences. Proc. Fifth Berkeley Symp. Math. Statist. Prob. 2, 351-372, University of California Press. MR 37:3835

5. Etemadi, N. (1981). An elementary proof of the strong law of large numbers. Z. Wahrscheinlichkeitstheor. Verw. Geb. 55, 119-122. MR 82b:60027

6. Giné, E., Hahn, G. and Zinn, J. (1983). Limit theorems for random sets. Springer Lecture notes in Math. 990, 112-135. MR 85d:60019 
7. Hoffmann-Jørgensen, J. (1985). The law of large numbers for non-measurable and nonseparable random elements. Colloque en l'honneur de L. Schwartz, pp. 299-356, Astérisque Vol. 131. Hermann, Paris 1985. MR 88a:60014

8. Klement, E.P., Puri, M.L. and Ralescu, D.A. (1986). Limit theorems for fuzzy random variables, Proc. R. Soc. Lond. A 407, 171-182. MR 88b:60092

9. Kruse, R. (1982). The strong law of large numbers for fuzzy random variables. Inform. Sci. 28, 233-241. MR 84k:60046

10. Proske, F.N. (1997). Limit theorems for fuzzy random variables. Ph.D. Thesis, Ulm University, Germany

11. Proske, F.N. and Puri, M.L. (2002). Central Limit Theorem for Banach Space valued fuzzy random variables. Proc. Amer. Math. Soc. 130, 1493-1501.

12. Puri, M.L. and Ralescu, D.A. (1983). Differentials of fuzzy functions, J. Math. Anal. Appl. 91, 552-558. MR 85c:26014

13. (1985). Limit theorems for random compact sets in Banach spaces, Math. Proc. Cam. Phil. Soc. 97, 151-158. MR 86c:60024

14. (1986). Fuzzy random variables. J. Math. Anal. Appl. 114, 409-422. MR 87f:03159

15. Talagrand, M. (1987). The Glivenko-Cantelli problem. Ann. Prob. 15, 837-870. MR 88h:60012

16. Zadeh, L.A. (1965). Fuzzy Sets, Information and Control 8, 338-353. MR 36:2509

Abt. Math. III, Universität Ulm, 89069 Ulm, Germany

E-mail address: frproske@metronet.de

Current address: Department of Mathematics, University of Oslo, 1053 Blindern, 0316 Oslo, Norway

E-mail address: proske@math.uio.no

Department of Mathematics, Indiana University, Bloomington, Indiana 47405

E-mail address: puri@indiana.edu 